\title{
IX. Further contributions to non-metrical vector algebra
}

\section{Silberstein Ph.D.}

To cite this article: L. Silberstein Ph.D. (1919) IX. Further contributions to non-metrical vector algebra, Philosophical Magazine Series 6, 38:223, 115-143, DOI: 10.1080/14786440708635934

To link to this article: http://dx.doi.org/10.1080/14786440708635934

曲 Published online: 08 Apr 2009.

Submit your article to this journal ๘

Џ Article views: 4

Q View related articles $\sqsubset$ 
of $\mathrm{R}$ being a pure convention. If, however, the equations of motion can be put in the canonical form, there is a definite reason of convenience for taking $R=1$; for this is the only value that makes the "force" the gradient of a scalar potential not involving the velocities explicitly. Thus the force is defined as $\left(f_{x}, f_{y}, f_{z}\right)$ and the energy * as $\frac{1}{2} \mu c^{2} \gamma^{2}$. The "mass" similarly becomes $\mu \gamma^{2}$.

4. In accordance with the theorem first quoted we have

$$
\begin{aligned}
\delta \int \mathrm{L} d \tau & =0, . \\
\mathrm{L} & =p_{x} \frac{d v}{d \tau}+p_{y} \frac{d y}{d \tau}+p_{z} \frac{d z}{d \tau}+p_{u} \frac{d u}{d \tau}-\mathrm{H} \\
& =\mathrm{U}+\text { constant. . . . . . . . . . . . }
\end{aligned}
$$

where

Thus the only possible form of $\mathrm{L}$ is that obtained by Silberstein, namely a constant + a scalar independent of the velocities, which is itself the force-function from which the force is derived.

IX. Further Contributions to Non-Metrical Vector Algebra. By L. Silberstein, Ph.D., Lecturer in Mathematical Physics at the University of Rome $\dagger$.

1. TN a recently published little book $\ddagger$ I havo endeavoured to build up a simple vector algebra independent of the parallel-axiom and of the axioms of congruence, and therefore of any idea of measurement by means of "rigid" bodies. This algebra consisted in essence only of addition and equality of vectors. Notwithstanding this poverty of means the said algebra was shown to be sufficient for the treatment of any purely geometric (i.e. projective) problems; for any such problem is ultimately concerned with crosses of straights, joins of points, with concurrency, collinearity, and similar questions, and all of these can be covered by the sole operation of addition, with its inverse and its iterations. No urgent need, therefore, was felt for the introduction of a "multiplication" of vectors by vectors. Also, at the time of writing and up to the last moment of

* This possibility of this value of the energy is indicated by Dr. Wilson (loc. cit.).

$\dagger$ Communicated by Prof. A. N. Whitehead, F.R.S.

I 'Projective Vector Algebra,' an algebra of vectors independent or the axioms of congruence and of parallels. Pp. 78. Bell \& Sons London, 1919 (May). 


\section{Dr. L. Silberstein : Further Contributions}

publishing of that book, no reasonably simple multiplicative. operation, independent of congruence and of parallels, presented itself to the writer. During the reading of the proofsheets, however, a not too-complicated non-metrical generalization of the scalar product, and then also of the vector product, of two vectors spontaneously suggested itself. And since, for technical reasons, this theory of vector multiplication came too late to be included in the said volume, it seemed worth while to publish it separately in the present paper which, to facilitate its perusal, will be kept uniform with the treatment (including nomenclature and symbolism) adopted in 'Projective Vector Algebra.' In order to avoid covering unnecessarily many pages of this Journal, the definitions and theorems concerning vector-equality and addition with their immediate consequences will not be repeated here. As often as the need arises the reader will be referred to the said book, which he will be assumed to have read or at least perused. It will be shortly referred to as $P . V$.A., with the section number whenever necessary.

Here but one more remark to justify this paper. As was said a moment ago vector addition is sufficient by itself to cover the field of projective geometry, $i$. $e$. to treat its problems algebraically. Thus, strictly speaking, vector "nultiplication" would be a superfuous operation. So, in fact, it is in a certain sense. But "multiplication" enables us to treat many of those problems in a considerably simpler manner. The multiplication of vectors by vectors, especially if it is so defined as to be distributive, is a powerful instrument of algebraical investigation, and therefore a very desirable supplement of addition.

2. Standard or Unit Conic.-It will be well to treat first the relations in a plane. The passage to three-dimensional space (section 6) will offer no difficulties.

In P.V.A. the concept of equality was applied to such pairs of vectors only as have different origins, while vectors emanating from a common origin were* complete strangers to one another, no relation between them having been dofined, unless one counts their distinctness. This distinctness consisted in their being on distinct lines (straights), and therefore in having different "termini" or T-points, their crosses with the conventionally fixed straight, the $T$-line. In more familiar language, our co-initial vectors differ in " direction," and since their "lengths" or "sizes" as tested by "rigid transferers" are entirely foreign to our circle of ideas, all these vectors have among themselves no feature in common.

* With the only exception of a vector $O A$ and its negative $O A^{\prime}=$ $-(0.1)$. 
Such being the case we are free to fix the standard or anit vectors, say $a, b, c, d$, etc. upon the rays emanating from their common origin $O$ quite at our pleasure, choosing them conventionally and independently of one another. There is but one limitation to this freedom, viz. if the ray $l^{\prime}$ is the continuation of the ray $l$, and if $a=0 a$ has been chosen as the unit vector for $l$, the unit vector $a^{\prime}=\left(a^{\prime}\right.$ for $l^{\prime}$ is to be such that, with reference to the fixed T-line,

$$
\mathbf{a}^{\prime}=-\mathbf{a} \text {. }
$$

This means that, if $T_{a}$ be the terminus of the straight $\left(l^{\prime} O l\right)$ in question, $a^{\prime}, O, a, T_{a}$ should be a harmonic range, with $a^{\prime}, a$ as conjugates.

Keeping this only condition in mind let us draw round $O$ some closed continuous curve in order to standardize at once the whole pencil of our vectors. Such a curve could be drawn in a variety of ways. It will be remembered, however, that its only office is to enable us to describe numerically the geometrical properties of the plane in as convenient a manner as possible. Now, with this aim in view, it will be found particularly convenient to choose as such standard curve a conic which, as is well-known, can be generated by purely projective processes.

Let, therefore, $O$, the origin of all our vectors, be surrounded by some conventionally fixed closed ${ }^{*}$ conic (ellipse) which we will call the stundard or the unit conir, and which will be denoted by $\kappa$.

Suppose the T-line has not yet been selected. Then by the very act of surrounding $O$ with the conic $\kappa$ and declaring it to be our unit conic (i.e. such that all its radii vectores $O_{a}$, $O b$, etc. are unit vectors) the $T$-line is co-determined. In fact, any secant $a^{\prime} O a$ drawn through $O$, which will hereafter be called a diameter of $\kappa$, meets the required T-line in a point $T_{a}$ which is the fourth barmonic to $a^{\prime}, \theta, a$, conjugate to $O$. In short, the $T$-line to match the standard conic is the polar of $O$ with respect to $\kappa$. Thus, having drawn $\kappa$, draw through $O$ (which will be called the centre of $\kappa$ ) any two diameters $a a^{\prime}$ and $b b^{\prime}$ (fig. 1); let $a b$ and $b^{\prime} a^{\prime}$ cross in $M$, and $b^{\prime} a$ and $a^{\prime} b$ in $N$; then the join $M N$ will be the required $T$-line, supplementing our reterence system. The origin $O$ being within the conic, its polar, the $T$-line, will lie outside $\kappa$.

We shall denote all unit vectors, such as $O a$ or $O b$, by small clarendons, a, b, etc., using capitals, A, B, etc. for any

* I. e. such that no tangents can be drawn from $O$ to the conic. 
vectors on the lines of $\mathbf{a}, \mathbf{b}$, etc. Any vector $O A=\mathbf{A}$ can be written $A=\sigma a$, where $\sigma$ is a scalar number which can always be made positive (by choosing appropriately between a and

Fig. 1.

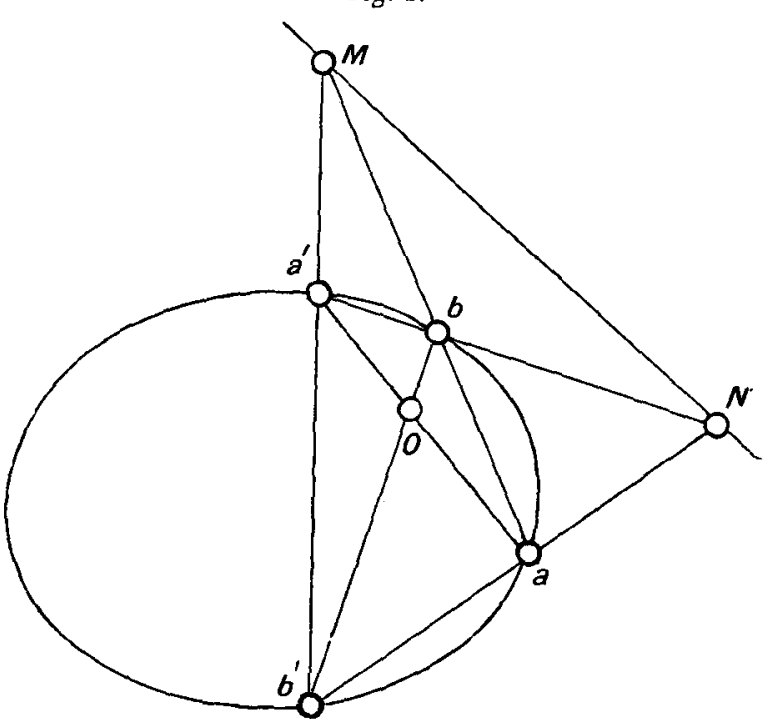

its negative $a^{\prime}$ ). With this understanding we will denote $\sigma$ by $|\mathbf{A}|$, so that

$$
\mathbf{A}=\mathbf{A} \mid \mathbf{a},
$$

and we will call $|\mathbf{A}|$ the tensor of $\mathbf{A}$, always of course with respect to the chosen $\kappa$ and $T$. Sometimes $A$ (where it cannot be confounded with "the point $A$ ") will be used as a short for $\mathbf{A}$. With this notation the equation of the standard conic will be

$$
|\mathbf{r}|=1 \text {, . . . . . . . ( }(\kappa) \text {. }
$$

where $\mathbf{r}$ is any vector drawn from $O$ to the conic $\boldsymbol{\kappa}$.

Having thus fixed upon every line through the origin the points 0,1 and os ( $T$-point), we can construct upon it in the well-known way (P.V.A., 6,7 ) the points 2,3 , etc., $\frac{1}{2}, \frac{1}{3}$, etc., in fact, the whole projective scale. The locus of the end-points of all vectors such as $2 a, 2 b$, etc. will again be a conic, $\kappa_{2}$ say, whose equation will be

and so on, in general,

$$
|\mathbf{r}|=2 \text {, }
$$

$$
|\mathbf{r}|=r=\text { const. }
$$


will again be a conic, $\kappa_{r}$, homologous with the standard conic. For $0<r<1$, all these conics will be closed and contained within $x$. For $r>1$, however, these conics will not continue for ever to be closed.

When $r$ reaches a certain value, say $r_{p}, k_{p}$ will be a parabola, and for $r \geq r_{p}$ the conic will either at once split into two branches, a new branch appearing "beyond" the $T$-line, or else we shall continue to have onebranched curves up to a certain value $r_{h}>r_{p}$, and only when $r_{h}$ is exceeded, two-branched conies (hyperbolæ). The former will be the case for the euclidean, and the latter for a lobatchevskyan space. (This, behaviour is closely connected with the peculiarities of the construction of the negative of a vector, $c f$. P.V.A. 9.) But details of this kind being irrelevant for our purposes, need not detain us here. For $r \rightarrow \infty$ the conic $\kappa_{r}$. will tend to a pair of straight lines falling, so to spealk, into. the T-line from both sides. (If space is elliptic, the T-line, as every straight, has but one "side"; in such cases, as in P.V.A., let us contemplate a "restricted region" broad enough to embrace all the contemplated figures.)

Let $x, y$ be the (non-homogeneous) projective coordinates of a point of $\kappa$ with respect to $O$ as origin and $a, b$ as axes, so that

$$
\mathbf{r}=x \mathbf{a}+y \mathbf{b},
$$

which reads : the end-point of $\mathbf{r}$ is reached from $O$ by $x$ projective unit steps along the a-line, followed by $y$ steps along $\mathbf{b}$, or vice versa (addition being commutative). Then, remembering that $|\mathbf{a}|$ is to be the unit of $x$, and $|\mathbf{b}|$ that of $y$, the equation of our standard conic with respect to any axes a, b whatever, can be written

$$
x^{2}+y^{2}+2 c_{12} x y=1 \text {. }
$$

The value of the coefficient $c_{12}$ will depend upon the choice of the particular pair $\mathbf{a}, \mathbf{b}$ as axes. It will be characteristic for the pair of rays represented by $\mathrm{a}, \mathrm{b}$ or, if we prefer to put it so, for the "angle a, b." To bring this into evidence let us better introduce, instead of $c_{12}$, the symbol

$$
\text { (ab) or (ba), }
$$

indiscriminately, since the rôles of $a$ and of $b$ in making up the scalar number $c_{12}$ are manifestly the same. Both (ab) and (ba) are hitherto mere synonyms of $c_{12}$, the coefficient of $2 x y$, and nothing else. After this warning we can write, for the standard conic,

$$
x^{2}+y^{2}+2(\mathrm{ab}) x y=1, \text {. . . . . }
$$

with respect to any (distinct) $a, b$ as axes. How to unravel 
the properties of (ab) from this somewhat implicit definition, will be shown presently.

Meanwhile, in order to be able to treat vectors with any origin other than $O$, let us recall from P.V.A. (section 8) the definition according to which all equal vectors are, in the first place, co-terminal, i. e. concurrent with one another in a $T$-point. 'Thus, if $T_{a}$ be the terminus of $\mathrm{a}=O \alpha$, and it is required to construct, with any given $O^{\prime}$ as origin (not on $T)$, the vector equal to a, we have first of all to draw $O^{\prime} T_{a}$; next, to obtain its end-point, we have to draw $O O^{\prime}$ crossing the $T$-line in $T^{\prime}$, say; then the straight $a T^{\prime}$ will cross $O^{\prime} T_{a}$ in $a^{\prime}$, the end-point of the required vector. Having thus obtained, on the line $O^{\prime} T_{a}$, the unit, we can in the wellknown way, construct upon it the vectors equal to $2 \mathbf{a}, 3 \mathbf{a}$, and so on, in general $O^{\prime} A^{\prime}=\mathbf{A}^{\prime}=\left|\mathbf{A}^{\prime}\right|$ a. This enables us to speak in a perfectly definite sense of the tensor of a vector drawn anywhere on the contemplated plane, as of the number of equal projective (unit) steps contained in it. Similarly we can construct, from $U^{\prime}$, vectors equal to $\mathbf{b}=O b, \mathbf{c}=O c$, etc., and thus reproduce (not to say "transfer") the original standard $\kappa$ at any spot where it is desired * Notice that if $\kappa^{\prime}$ be such a reproduction of the standard round $(\nu$, the original T-line will again be the polar of $O^{\prime}$ with respect to $\kappa^{\prime}$. We thus see that our original $O$ is by no means a privileged point of the contemplated two-space. Any $O^{\prime}$ is as good a point. The 'T-line, however, does play a privileged rôle, being the common polar of the "centres" of all these standard conics with respect to each of them. It is also the polar of $O^{\prime}$ with respect to any $\kappa_{r}{ }^{\prime}$, the homologue of $\kappa^{\prime}$.

But it will be remembered that even the $T$-line, although fixed for the duration of an investigation, is but an arbitrury reference line, and can, in different instances, be chosen in a manner most suitable for the investigation of the given figure or figures drawn on the plane, the only geometrical entities. How the choice of this reference line influences the position of the "centres" of the standard conics $\kappa^{\prime}$ we already know ; each of them is the pole of $T$ with respect to $\kappa^{\prime}$.

* The physicist will notice the difference between the standard $\kappa$ and, say, the metre standard preserved at Paris. It is not enough to make a copy of the latter at the spot, in Paris, it has to be carried about; the former, however, is "reproduced" or constructed at the place where it is just required. This is a capital difference. Moreover, it is enough to construct a few isolated points of $x^{\prime}$. For, as is well known, five points of $\kappa^{\prime}$ would (even in the absence of $T$ ) suffice to construct, without further appeal to $k$, any number of other points of $\kappa^{\prime}$. 
3. Geometrical meaning of (ab). Definition of Orthogonality.-In order to perceive clearly the intrinsic nature of the ordinary number (ab) or (ba) as a property of the vector pair, a, b, let us construct graphically some vector expression into which (ab) will enter alongside with things already known to us. For this purpose let us return to the equation (1) of the standard conic $\kappa$. From this we find easily, as the equation of the tangent to $\kappa$ at any of its points $\bar{x}, \bar{y}$,

$$
[a+(\mathbf{a b}) y] x+[y+(\mathbf{a b}) x] \bar{y}=1,
$$

$x, y$ being the coordinates of any point of the tangent line. In particular, the tangents at the end-points $a(\bar{x}=1, y=0)$ and $b(\vec{y}=1, \vec{x}=0)$ of the vectors $a, b$ used as axes will have the equations

$$
\begin{aligned}
& w+(\mathrm{ab}) y=1, . . \quad . \quad . \quad . \quad\left(t_{\mathrm{a}}\right) \\
& \text { (ab) } x+y=1 \text {. . . . . . . }
\end{aligned}
$$

Let $P$ be the cross of the two tangents $t_{a}, t_{b}$, or the pole of the chord $a b$ as polar. Then its coordinates, the solutions of the last two equations, will be

$$
\xi=\eta=\begin{gathered}
1 \\
1+(\mathrm{ab})
\end{gathered}
$$

and the vector $\mathrm{P}=O P$, which is $\xi \mathrm{a}+\eta \mathrm{b}$, will become

$$
\mathbf{P}=\frac{\mathbf{S}}{1+(\mathbf{a b})}, \text { where } \mathbf{S}=\mathbf{a}+\mathbf{b} \text {. }
$$

It will be remembered that $S$, the end-point of $\mathbf{S}$, is the cross of $a T_{b}$ with $b T_{a}$ (P.V.A., 4).

This is the required expression, or equation, since it contains, besides ( $\mathbf{a b}$ ), only $\mathbf{P}$ and $\mathbf{S}$, well determined and easily constructible things. Hitherto (ab) was defined only as the coefficient of $2 x y$ in the coordinate equation of $\kappa$ with $a, b$ as axes. Henceforth we can drop that equation and consider (ab) as defined by the vector equation (2), which shows at once the intrinsic nature of $(\mathbf{a b})$. Such being the case we can, on the other hand, expand (ab) using, for instance, the components of $\mathbf{a}$ and $\mathbf{b}$ along some auxiliary axes; for we shall then know that the value thus obtained is independent of these auxiliaries. We may profit from this possibility later on.

Meanwhile let us concentrate our attention upon the last equation. It will teach us many interesting things. First of all, $1+(\mathrm{ab})$ being a mere number, it shows us that $S$ is 
collinear with $O$ and $P$ (fig. 2). In other words, the vectors. $\mathbf{P}, \mathbf{s}$ differ only through their tensor. Such being the case

Fig. 2.

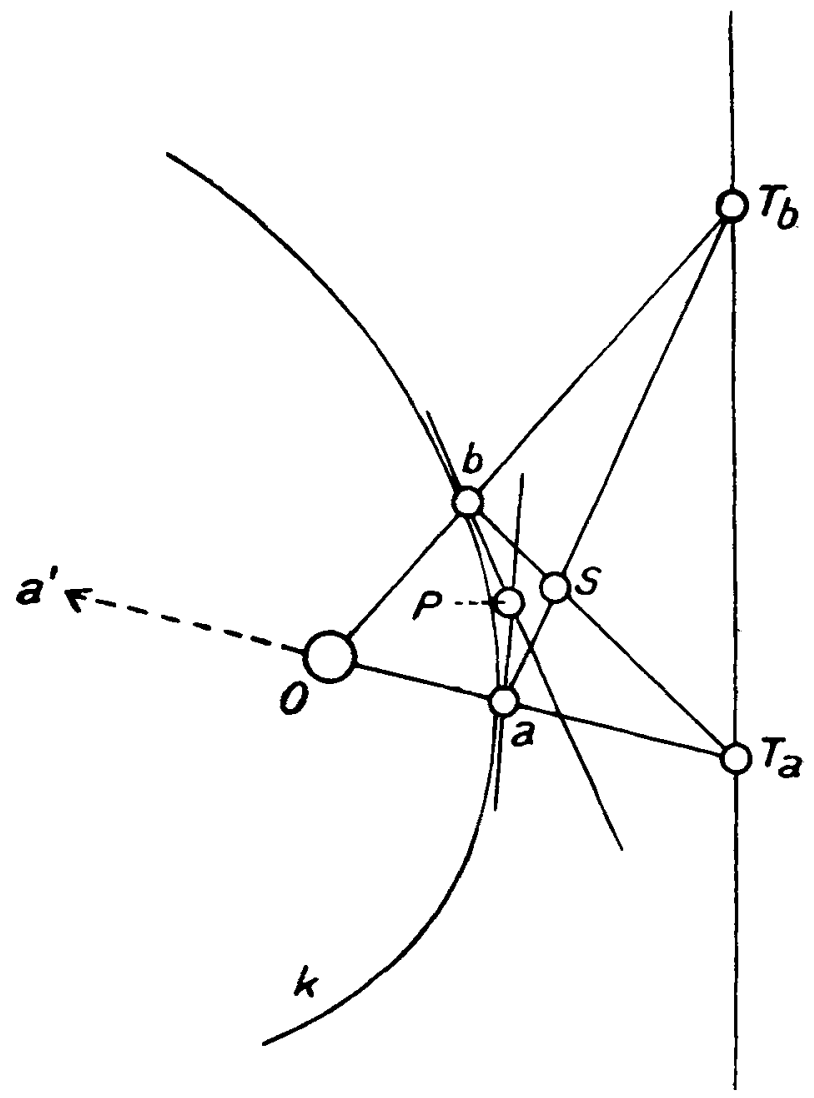

there is no objection against writing (2) in the form $\mathbf{l}+(\mathrm{ab})=\mathbf{S} / \mathbf{P}$, or also

$$
(\mathbf{a b})=\frac{\mathbf{s}-\mathbf{P}}{\mathbf{P}}=\frac{O S-O P}{U P}, . .
$$

In the next place we see from this equation (and from the drawing) that when $\mathrm{b}=\mathrm{a}, i$. $e$. when $a, b$ coalesce, and therefore $S=2 a$, while $P=a$, we have

$$
\text { (aa) }=1 \text {, }
$$

for any unit vector $a$. Similarly if $b=a^{\prime} \equiv-a$, the vector $\mathbf{S}$ 
vanishes, and

$$
\left(a a^{\prime}\right)=-1 \text {. }
$$

These values correspond to what way be called the "zero angle," and the "straight angle," respectively,-using these names only as synonyms of the pairs $(a, a)$ and $\left(a, a^{\prime}\right)$. For hitherto we have not introduced the general concept of "angle" as a magnitude.

But the most important thing for our present purpose is to note that when

$$
\mathbf{P}=\mathbf{S} \equiv \mathbf{a}+\mathbf{b},
$$

that is to say, when the pole of ab coincides with the cross of $a T_{b}$ and $b T_{a}$, our equation gives

$$
(\mathbf{a b})=0 \text {. }
$$

I propose to call such, manifestly remarkable, pairs a, b normal or orthogonal, or perpendicular vectors. Their definition can be conveniently written

$$
\mathbf{P}_{a b}=\mathbf{a}+\mathbf{b} \text {. }
$$

the double suffix reminding us that $P$ is the pole of the chord $a b$. It will be noticed that, when used as coordinate axes, any such pair $a, b$ reduces the standard-conic equation to

$$
x^{2}+y^{2}=1 \text {. . . . . . . . }
$$

Fig. 3.

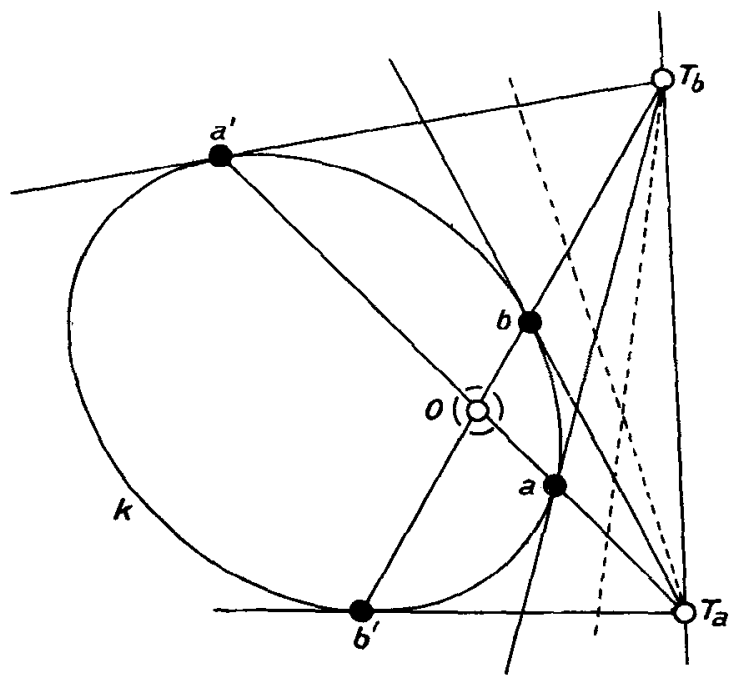

Any number of such orthogonal pairs can at once be constructed. In fact, from the form (4) of their definition we see that both $T_{a}^{b}$ and $T_{b} a$ are tangents to $\kappa$. Thus, if any $a=O a$ (fig. 3) be given, prolong it up to its terminus $T_{a}$. 
and draw from $T_{a}$ a tangent to $\kappa$. Then the contact point $b$ will be the end-point of the required unit vector $b$ normal to a. The proof that $T_{b} a$ will then also be a tangent may be left to the reader. Again, the other tangent from $T_{a}$ will touch $\kappa$ in $b^{\prime}$, which will be found to be collinear with $b, O$, so that $\mathbf{b}^{\prime}=-\mathbf{b}$. Similarly, the other tangent from $T_{b}$ will touch $\kappa$ in $a^{\prime}$ the end-point of $-a$. Thus, together with $O a, O b$ also $O a, O b^{\prime}$ and $O a^{\prime}, O b$ wiil become automatically pairs of orthogonal rays. In short, the straights $a a^{\prime}$ and $b b^{\prime}$ will be orthogonal. Since $T_{a}$ is the pole of $b b^{\prime}$, and $T_{b}$ that of $a a^{\prime}$, we see that our orthogonal lines are, in usual nomenclature, pairs of conjugate lines with respect to $\kappa$. At the same time we see that, in technical language, $O T_{a} T_{b}$ will be a self-polar triangle with respect to $\kappa$.

We thus know how to draw to any straight passing through $O$ a perpendicular in $O$. This limitation to the origin can be easily removed. In fact, let us generalize our original definition by postulating that if $(a b)=0$, and if $\mathbf{v}$ be any vector equal to $b$, then also

$$
\text { (av) }=0 \text {; }
$$

more generally, if $\mathbf{a}^{\prime}=\mathbf{a}$ and $\mathbf{b}^{\prime}=\mathbf{b}$, then $\left(\mathrm{a}^{\prime} \mathbf{b}^{\prime}\right)=(\mathbf{a} b)$. Now, all vectors equal to a are coterminal with a. Thus, returning to fig. 3, all lines of the pencil centred at $T_{i}$ will be orthogonal to all those of the pencil centred at $T_{a}$. In particular, the tangent to $\kappa$ at any of its points (as $T_{b} a$ ) will be normal to the corresponding diameter $\left(a^{\prime}(\iota)\right.$ *.

This shows us also that the proper representatives of the orthogonality thus defined are the pairs of corresponding pencil-centres themselves, each pair being so correlated as $T_{a}, T_{b}$ in fig. 3. (These pairs of points are, in common nomenclature, "conjugate" "with respect to the conic $\kappa$.) We may have an opportunity to return to similar questions. Meanwhile let us proceed with our chief subject.

4. Scalar Product defined. Distributivity. Let $\mathbf{A}=|\mathbf{A}| \mathbf{a}$ and $\mathbf{B}=|\mathbf{B}| \mathbf{b}$ be any two vectors.

Let us denote by (AB), and call the scalar product of $\mathbf{A}$ into $B$, the number

$$
(\mathbf{A B})=|\mathbf{A}| \cdot|\mathbf{B}|(\mathbf{a b})
$$

Since the tensors are ordinary numbers and $(\mathbf{a b})=(\mathbf{b a})$, we have also

$$
(\mathbf{A B})=(\mathbf{B A}) \text {, }
$$

the commutative property.

* Notice in passing that the $T$-line itself will be orthogonal to every other straight line whatever. 
If $m, n$ be any scalars, then $|m \mathbf{A}|=m|\mathbf{A}|,|n \mathbf{B}|=n|\mathbf{B}|$, so that, by the definition (6), the product of $m \mathbf{A}$ into $n \mathbf{B}$ is equal to $m n$ times $(\mathbf{A B})$. In view of this property we can henceforth drop the brackets and write the scalar product simply $\mathbf{A B}$ and, instead of (6),

$$
\mathbf{A B}=|\mathbf{A}| \cdot|\mathbf{B}| \mathbf{a b}
$$

If $\mathbf{A}, \mathbf{B}$ are orthogonal, $i$. e. if $\mathbf{a b}=0$, we have $\mathbf{A B}=0$ unless, of course, A or B are infinite or T-vectors.

We have already seen that $a=1$, for any unit vector $a$; instead of this we shall write $\mathbf{a}^{2}=1$. Thus, for any vector $\mathbf{A}$,

$$
\mathbf{A}^{2}=|\mathbf{A}|^{2}=A^{2} \text {. . . . . . . . }
$$

In words, the scalar autoproduct of any vector is equal to the square of its tensor, exactly as in ordinary, euclidean, vector algebra.

But what makes our generalized scalar multiplication of vectors a powerful operation is the fact that it shares with the ordinary one the property of distrilutivity. This can be proved in a variety of ways. The shortest of these seems to be the following one.

Fig. 4.

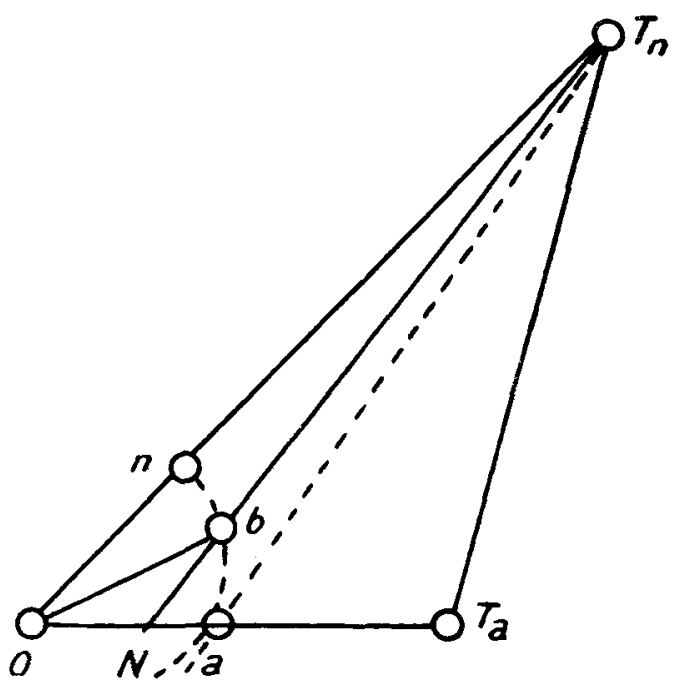

By (b) or (6a) the definition of $\mathbf{A B}$ is reduced to that of $a b$, given originally by (1). In the preceding Section we have already given a geometrical representation of this number. Another, still simpler representation is obtained by drawing from $b$ the perpendicular to a. Let $\mathbf{n}$ (fig. 4) be 
normal to a, and let the terminus of $\mathrm{n}$ be $T_{n}$ (this is also obtained by simply drawing the tangent at $a$ ). Draw $T_{n} b$ crossing the a-line in $N$. Then the a-coordinate of $N$ will be ab or, vectorially,

$$
O N=(\mathbf{a b}) \mathbf{a} .
$$

To see this, take the orthogonal pair $\mathbf{a}, \mathfrak{n}$ as axes of $x, y$, so that the equation of $\kappa$ will be

$$
x^{2}+y^{2}=1 \text {. }
$$
have

Now, if $\alpha, v$ are the coordinates of $b$ with these axes, we

$$
\mathrm{b}=\alpha \mathrm{a}+\nu \mathrm{n}, \alpha^{2}+\nu^{2}=1 .
$$

Thus, if $x^{\prime}, y^{\prime}$ be the coordinates of any point $(x, y)$ with respect to $a, b$ as axes, we have

whence

$$
x \mathbf{a}+y \mathbf{n}=x^{\prime} \mathbf{a}+y^{\prime} \mathbf{b}=\left(x^{\prime}+\alpha y^{\prime}\right) \mathbf{a}+\nu y^{\prime} \mathbf{n},
$$

$$
x=x^{\prime}+\alpha y^{\prime} ; y=\nu y^{\prime},
$$

and the conic equation with $a, b$ as axes becomes

$$
x^{\prime 2}+y^{\prime 2}+2 \alpha x^{\prime} y^{\prime}=1 \text {. }
$$

Therefore, by the original definition of $a b$, as given in (1),

$$
\mathrm{ab}=\alpha,
$$

which is the a-coordinate of $b, i . e$. that of $N$. This proves the statement made in ( 8 ).

Thus $a b$ is obtained by the orthogonal projection of $b$ upon a or, similarly, by that of a upon $b$, exactly as in ordinary vector algebra, notwithstanding the different, more general meaning of our concepts.

Now, such being the case, the distributivity of the scalar product follows at once. In fact, if $\mathbf{A}, \mathbf{B}, \mathbf{C}$ be any vectors and $\mathbf{B}+\mathbf{C}=\mathbf{S}$, we have, first of all,

$$
\mathbf{A}(\mathbf{B}+\mathbf{C})=|\mathbf{A}| \cdot \mathbf{a S} \text {. }
$$

Now, whatever B, C, we can always reduce them to a chain, so that $\mathbf{S}$ will be the vector drawn from the origin of $\mathbf{B}$ to the end-point of $\mathbf{C}$. But the orthogonal projection of $\mathbf{S}$ upon $\mathbf{a}$ is instantly seen to be equal to the algebraic sum of the orthogonal projections of B and of C. Cf. fig. 5, in which $T_{n}$ is the $T$-point of the vector $n$ normal to $a$. Notice that the intercepts of the three perpendiculars will 
not re-cross one another; for they already crossed one another in $T_{n}$.

Fig. 5.

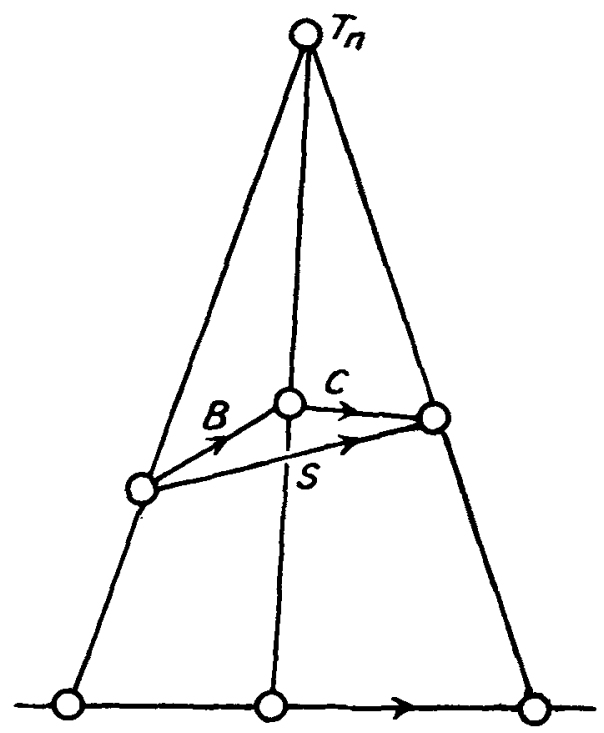

Thus we have the announced distributive property,

$$
\mathbf{A}(\mathbf{B}+\mathbf{C})=\mathbf{A B}+\mathbf{A C} \text {. }
$$

And since the commutative law holds as well, we have also

$$
(A+B)(C+D)=A C+A D+B C+B D
$$

and so on.

In fine, all ordinary rules hold for the generalized scalar product of vectors, as defined ahove by means of the standard conic.

The far-reaching geometrical consequences of this simple property are obvious. Every formuli known from euclidean geometry (or "trigonometry") will continue to hold, with the only difference that tensor or "size" of a vector will not stand for the number of centimetres or inches contained in it, but for the number of equal projective steps witl reference to the conventional unit-conic $\kappa$ and $T$-line, and that right angles (ortbogonality), equal angles, etc., have in our case a different, more general meaning. 
Thus for instance, if $\mathbf{A}$ is normal to $\mathbf{B}$, and if $\mathbf{C}=\mathbf{A}-\mathbf{B}$, we have, by the distributive law,

$$
\mathbf{C}^{2}=\mathbf{A}^{2}+\mathbf{B}^{2}
$$

or

$$
|\mathbf{C}|^{2}=|\mathbf{A}|^{2}+|\mathbf{B}|^{2},
$$

the generalization of Pythagoras' theorem, which reads : the squared number of projective unit steps contained in the lyypotenuse is equal to the sum of those contained in the sides.

The reader will find it a uzeful recreation to verify this graphically in a convenient case, such as $|\mathbf{A}|=3,|\mathbf{B}|=4$, drawing any ellipse as $\kappa$ round $O$, constructing its polar $T$, and so on. If he draws the rather numerous lines, necessary for the whole construction, with some care he will find the number of unit steps contained in $\mathbf{C}$ to be hardly distinguishable from 5. The writer has actually constructed this case with good success, his naive aim being originally to verify thus the analytic result.

More generally, for any triangle with $\mathbf{A}, \mathbf{B}$ and $\mathbf{C}=\mathbf{A}-\mathbf{B}$ as vector sides,

$$
|\mathbf{C}|^{2}=|\mathbf{A}|^{2}+|\mathbf{B}|^{2}-2|\mathbf{A}| \cdot|\mathbf{B}| \cdot \mathbf{a b} . \quad . \quad
$$

Many other examples of generalized theorems could at once be given, but those quoted above will suffice for the present.

5. Angles, their arc-measure and trigonometric functions.We have incidentally called the pair $\mathbf{a}, \mathrm{b}$ an "angle," in particular a, a a zero angle and a, - a a straight angle. We may now, without fear of a misunderstanding, call $a, b$, i. e. $a O b$ or $b O a$, in the case when $a b=0$, a right angle. In general, for any $a, b$, or their equals constructed anywhere, we can speak of the angle $a O b$. Still, it remains true that we do not yet know what "equal angles" and " multiples of an angle" mean, simply because we have not yet fixed the meaning of these words. There is nothing to prevent us. from denoting the angle $a O b$ by a single letter, say $\theta$, provided we keep in mind that hitherto this $\theta$ is not itself a magnitude. We shall define it presently as such.

Meanwhile we can speak of the number ab, already familiar to us, as a certuin "indirect measure" or "function" of the angle $\theta=a, b$. As such let us call it the cosine of $\theta$, writing

$$
a b=\cos \theta=\cos (a, b) .
$$

We know from Section 4 that this number is represented by the orthogonal projection $N$ of $b$ upon a, that is to say, that

$$
\cos \theta=\cos (\mathbf{a}, \mathbf{b})= \pm i O N \mid, \quad . \quad .
$$


according as $N$ falls on $O a$ or $O a^{\prime}$. We may, therefore, consider the latter equation as the definition of cosine. Similarly, let us define the sine of $\theta$ by the tensor of the normal $b N$, writing

$$
\sin \theta=\sin (\mathbf{a}, \mathbf{b})=|b N|, \quad . \quad . \quad .
$$

$a, b$ being points on the unit conic $\kappa$, as before. (The sine and the cosine of an angle $l O^{\prime} m$ constructed anywhere can at once be reduced to the above by joining the $T$-points of $O^{\prime} l, O^{\prime} m$ with $O$, and so on.) Using these familiar symbols, however, we must not yet confound them with the familiar trigonometrical functions, until we have learnt that they are identical with them. Such, in fact, tley will be shown to bo presently; and their analytical identity will become complete after an appropriate measure has been introduced for $\theta$, still to be defined as a magnitude.

At any rate we know already that if $\theta_{0}, \theta_{s}, \theta_{r}$ stand for the zero angle, the straight, and the right angle respectively, we have

$$
\cos \theta_{0}=1, \cos \theta_{s}=-1, \cos \theta_{r}=0, . .
$$

although we do not know, for example, what the numerical value of $\theta_{r}$ is. Again, since $O b N$ is a right-angled triangle and $O b$ is a unit vector, we have, by the generalized Pythagorean theorem, for any $\theta$,

$$
\sin ^{2} \theta+\cos ^{2} \theta=1 \text {. . . . . . }
$$

Moreover, again in virtue of the distributive property, we can deduce for our sin and $\cos$ the familiar additivity

Fig. $5 a$.

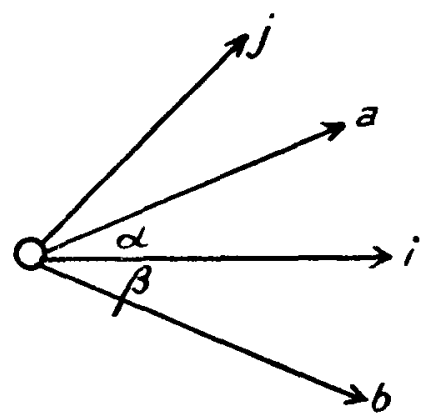

theorems. For this purpose it is enough to declare that if $\alpha=b O i$ and $\beta=a O i$ be co-vertical angles having the side $O i$ in common, $a O b$ is the sum of these angles; and sinilarly for the difference. In fact, if $i, a, b$ (fig. $5 a$ ) be the end-

Phil. Mag. S. 6. Vol. 38. No. 223. July 1919. 
points of the unit vectors $i, a, b$, and if the unit vector $j$ be normal to $i$, we have, by what has been explained previously,

$$
\begin{aligned}
& a=\mathbf{i} \cos \alpha+\mathbf{j} \sin \alpha, \\
& b=\mathbf{i} \cos \beta-\mathbf{j} \sin \beta,
\end{aligned}
$$

whence, by the distributive law of multiplication,

$$
\mathrm{ab}=\cos (\alpha+\beta)=\cos \alpha \cdot \cos \beta-\sin \alpha \cdot \sin \beta,
$$

whence also, by (12b), after easy transformations,

$$
\sin (\alpha+\beta)=\sin \alpha \cdot \cos \beta+\cos \alpha \cdot \sin \beta, .
$$

as in ordinary trigonometry.

Fig. 6.

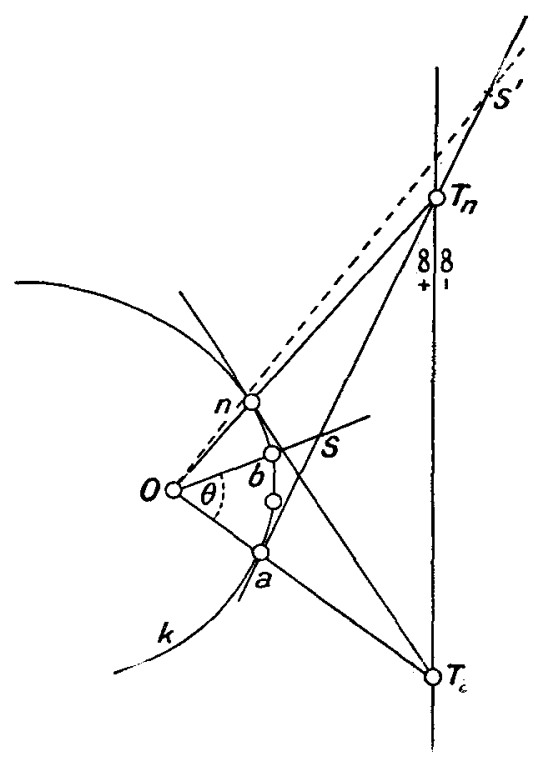

Leaving other details to the reader, let us still notice that $\tan \theta=\tan (a, b)$ can be defined either by

$$
\tan \theta=\sin \theta / \cos \theta, \text {. . . . }
$$

or graphically (fig. 6) by

$$
\tan \theta= \pm|a \mathbf{S}|
$$


where $S$ is the cross of the b-line with the conic tangent at $a$, the upper or the lower sign being used according as $S$ falls on the segment $a T_{n}$ or outside it (i. e. below $a$ or beyond $T_{n}$ ). It is not difficult to see that these two definitions are equivalent. In fact, if $\boldsymbol{n}$ be normal to a, and therefore co-terminal with $a S$, we have

$$
\mathrm{S}=\mathrm{a}+\lambda \mathrm{n}=\mu \mathrm{b}=\mu(\mathrm{a} \cos \theta+\mathrm{n} \sin \theta),
$$

whence $\mu \cos \theta=1, \mu \sin \theta=\lambda, \therefore \lambda=\sin \theta / \cos \theta$, and

$$
a S=\mathbf{S}-\mathbf{a}=\lambda \mathbf{n},
$$

so that

$$
\pm|a S|=\frac{\sin \theta}{\cos \theta}
$$

proving the statement.

Notice that the jump of $\tan \theta$ from $\infty$ to $-\infty$ when $\theta$ passes through a right angle $\left(e_{r}\right)$ obtains the following elegant representation: When $\mathbf{b}$ (fig. 6) approaches $\mathrm{n}$, the point $S$ approaches $T_{n}$, the value of $\tan \theta$ thus increasing to $+\infty$, and when $\mathrm{b}$ exceeds $\mathrm{n}$, the point $S$ passes across the $T$-line thus indicating the jump of $\tan \theta$ to $-\infty$. It will be remembered from the construction of the projective scale (P.V.A.) that, on every straight, to one side of $T$ corresponds $\infty$, and to the other side $-\infty$. The discussion of the obvious geometrical correlate of the fact that $\cos \theta$, for instance, does not exceed the values \pm 1 may be left to the reader. It will be enough for this purpose to note that the pencil of all lines $T_{n} b$ (leading to our previous $N$ ) is limited by the two tangents $T_{n} a, T_{n} a$.

Let us now pass to compare angles with one another, so as to be able to $\operatorname{treat} \theta$ as an algebraical magnitude. And to begin with let us give a definition of angle equality. This can, in the first place, be based upon $a b=\cos \theta$ itself.

Limiting ourselves to concave angles we may define two angles $\alpha$ and $\beta$ as equal if their cosines are equal, no matter where their vertices are situated *. In symbols, if

$$
\mathrm{ab}=\mathrm{Im}, \text {. . . . . . }
$$

we will say that angle $\mathbf{a}, \mathbf{b}=$ angle $\mathbf{~}, \mathbf{m}$.

The simplest sub-case of such a relation occurs when 1 and $a, m$ and $b$ are equal to one another but situated in different places. This gives, as a corollary of the above

* Notice that every angle having its vertex on the $T$-line (being of the form 1,1 ) is a zero angle, unless one of its sides is the $T$-line, when the angle is a right angle. Cf. supra. 
definition, the equality of all angles (such as $\alpha$ and $\beta$ in fig. 7) whose sides are coterminal. Such was the only case of angle comparison treated in P.V.A., Appendix A, where it was shown to be sufficient to prove that the non-metrical sum of

Fig. 7.

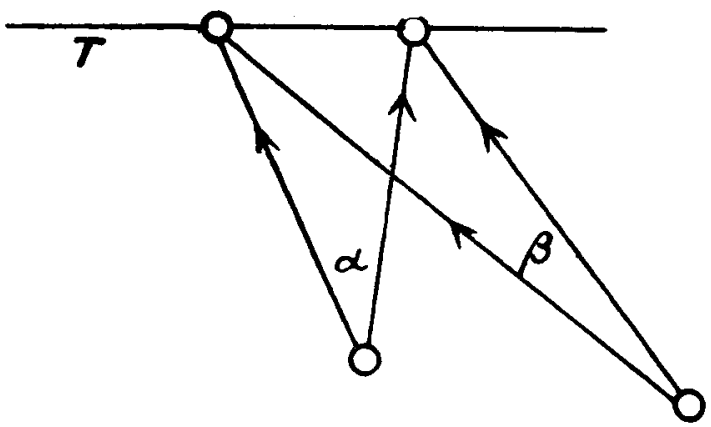

angles in every triangle (not traversed by the $T$-line nor having it as one of its sides) is equal to a straight angle or, as we now can say, to two right angles. We may return to this triangle property later on. Meanwhile let us proceed with the application of the definition (13) of angle equality to cases in which $1, \mathrm{~m}$ are vectoria ly different from $\mathbf{a}, \mathbf{b}$.

Without loss to generality we can assume all these four vectors to be co-initial in $O$, so that $a, b, l, m$ are on the conic $\kappa$. Let us put the question in the form of the problem : Given $\mathrm{a}, \mathrm{b}$, the sides of the angle $\alpha$, and given 1 , one side of the angle $\beta$, to construct $m$, so that

i. e. so that $\mathrm{lm}=a b$.

$$
\alpha=\beta \text {, }
$$

From $b$ draw the normal tr $O a$ meeting it in $N$; through $N$ imagine drawn the conic homologous to $\kappa$; let this cut $O l$ in $N^{\prime}$. Through $N^{\prime}$ draw the normal to 1 cutting $\kappa$ in two points; either of these will be the end-point of the required $\mathrm{m}$, giving $\beta=\alpha$. It is, for our purpose, by no means necessary to execute this construction in detail. (And a more speedy construction will be given in Section 9.) It is enough to contemplate it in order to see that, whatever 1 , an angle equal to $a$ can be constructed on either side of 1 , in a perfectly determined nanner.

In particular, if $l$ coincides with $b$, when the problem consists in the doubling of $\alpha$, the actual construction becomes a very simple process. For, involuntarily, we have already 
solved the inverse problem, viz. to bisect a given angle $a 0 \mathrm{~m}$. In fact, if $P$ be the pole of the chord am we have, by (2), and replacing $\mathrm{b}$ by $\mathrm{m}$,

$$
\mathbf{P}=\frac{\mathbf{a}+\mathbf{m}}{1+\mathbf{a m}} .
$$

Multiply this equation scalarly by a or by $\mathbf{m}$. The result will, in both cases, be 1 . Thus $\mathbf{P a}=\mathbf{P m}$, and therefore,

$$
\text { angle a, } \mathbf{P}=\text { angle } \mathbf{P}, \mathbf{m} \text {. }
$$

But $P$ and $S$, the end-point of $\mathbf{a}+\mathbf{m}$, are collinear with $O$. Thus also

$$
\text { aOS }=S O m,
$$

giving the required bisection of the angle $a O m$. Remembering that $S$ is the cross of $a T_{m}$ with $m T_{\alpha}$, this construction is obtained at once, as shown in fig. 8. (Notice in passing

\section{Fij. 8.}

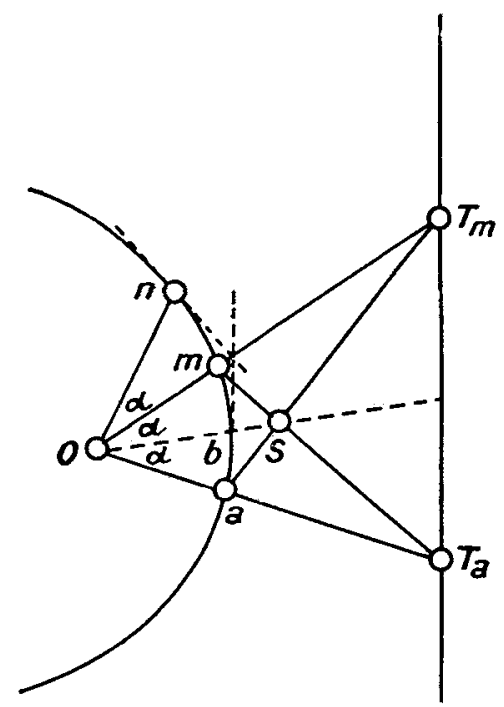

that $a S^{\prime}=0 m, O a=m S$, and that all four are unit vectors. Thus $\mathrm{OaS}_{m}$ is a generalized rhombus. In particular, if $a m=0$, it will be a generalized square.) Thus far the bisection of an angle. The solution of the inverse problem, originally required, follows at once by remembering that $P$, the pole of the required chord $a m$, is collinear with $O, S$. Thus to double $a O b$, draw the tangent at $a$, crossing the b-line in $P$; 
the second tangent from $P$ will give the required $m$ as its contact-point.

Proceeding in the same manner with $b, m$ as before with $a, b$, we can construct the point $n$, such that $a O_{n}=3 \alpha$, and so on.

Combining both processes we can, from a given $\alpha$, build up any angle

$$
\frac{m \alpha}{2^{n}}
$$

where $m, n$ are any positive integers.

Although the equality definition based on ab suffices for cumparing angles with one another, it is interesting to introduce another angle measure which we may call the arc-measure of an angle. It will again suffice to consider angles with vertex $O$. Let $a, b$ be a pair of points on the conic $\kappa$ determining, let us say, the concave angle $\mathbf{a}, \mathbf{b}=a \mathbf{O} b$. Draw a chain of vector chords $a p_{1}, p_{1} p_{2}$, etc., $p_{n} b$. Consider the sum of the tensors of all these vectors,

$$
\left|a p_{1}\right|+\left|p_{1} p_{2}\right|+\ldots+\left|p_{n} b\right|=\Sigma \Delta s, \text { say },
$$

and making the necessary analytical assumptions, pass to its limit

$$
s_{a b}=\int_{a}^{b_{b}} d s,
$$

which, if it exists, is obviously an ordinary scalar number, and call it the "length" of the arc or simply the are $\widehat{a b}$ of our conic. It is the number of projective unit steps contained in $\widehat{a b}$.

In particular jet $s_{r}$ stand for the are subtending a right angle (any right angle), i.e. corresponding to the case in which $a b=0$. What the numerical value of $s_{r}$ is will be seen presently.

Lot us now define the pure number

$$
\theta=s_{a b}
$$

as the arc-measure of the angle $\mathrm{a}, \mathrm{b}$ or $a \mathrm{O}$.

It is not difficult to see that two angles which are equal according to the previous definition (1.3) have also equal:arcmeasures. In fact, let

$$
\mathrm{lm}=\mathrm{ab}=x .
$$

Then, taking $\mathrm{m}$ as the $x$-axis of orthogonal coordinates $x, y$, 
we have, by the generalized Pythagorean theorem,

$$
\int_{l}^{m} d s=\left|\int_{1}^{x} \sqrt{1+(d y / d x)^{2}} \cdot d x\right|
$$

with $x^{2}+y^{2}=1$ as the conic equation. But taking a as the $\boldsymbol{x}$-axis of orthogonal coordinates $\boldsymbol{\xi}, \boldsymbol{\eta}$, we have again

$$
\int_{a}^{b} d s=\left|\int_{1}^{x} \sqrt{1+(d \eta / d \xi)^{2}} \cdot d \xi\right|, \xi^{2}+\eta^{2}=1 .
$$

Thus the measures of the two angles are equal. In short, the common arc-measure of both, and therefore of all (concave) angles having the $\operatorname{cosin} \theta a b=\cos \theta=x$ is given by

$$
\theta=-\int_{1}^{x} \frac{d x}{\sqrt{1-x^{2}}}
$$

which is the well-known function $\cos ^{-1} x$ or $\operatorname{arc} \cos x$. From this the converse theorem : if $\theta_{a b}=\theta_{l m}$ then $a b=l m$, follows at once. In particular, if $a b=0$, we shall have, as the arcmeasure of all right angles,

$$
\theta_{r}=\int_{0}^{1} \frac{d x}{\sqrt{1-x^{2}}}=\frac{\pi}{2},
$$

where $\pi$ is the familiar transcendental number $3 \cdot 14 \ldots$ Thus also the circumference of the unit conic $\kappa$ is seen to be $2 \pi, i$. e. to contain $2 \pi$ projective unit steps or-as I should like to propose to call them--2 $\pi$ staudtians (the projective scale being due to George von Staudt). This simple result holds, of course, independently of the particular shape of the ellipse adopted as standard conic and of the position of $O$ inside it.

We need not be surprised by this result, which is but one instance of the reappearance of familiar geometrical formulæ. For, as was already mentioned, the validity of the distributive law of multiplication of vectors will carry with itself a formal and numerical identity with all euclidean formulæ, the numbers of inches or of cms. being replaced by the numbers of staudtians. That the same thing should hold for curved ares (consldered as limits of polygons) as well as for tensors of vectors, or "sizes" of straight segments, might have been expected at once.

In order to make the angle $\theta$ liable of assuming positive and negative values it is enough to prescribe a sense of circulation round $\kappa$ as the positive one, and the opposite as the negative one, in much the same way as in the familiar treatment of angles as " rotations." 
The integration just applied to the standard conic can obviously be extended to the "rectification" of any curve determined by $\phi(x, y)=0$ or $y=f(x)$, the line-element, or tensor $d s$ of an infinitesimal vector being given by

$$
d s^{2}=d x^{2}+d y^{2}+2 \mathrm{ab} d x d y
$$

in case of any $a, b$ as coordinate axes, and by

$$
d s^{2}=d x^{2}+d y^{2} \text {. . . . . . }
$$

in case of orthogonal axes. This subject does not call for any further explanations. Nor does the proof that the are of any conic $\kappa_{r}, i$.e. $\mathrm{r}^{2}=r^{2}=$ const. (homologous with $\kappa$ ) subtending the angle $\theta$ is equal to

$$
\theta r
$$

offer any difficulties. Thus also the whole circumference of a conic $\kappa_{r}$ (or of its reproduction obtained anywhere by the transformation $\mathbf{r}^{\prime}=\mathbf{r}+$ const.) will be equal to

\section{$2 \pi r$ staudtians,}

no matter whether $\kappa$, is still an ellipse or has already become, say, a parabola (whose length would in the ordinary treatment be infinite). Hyperbolæe muy offer some peculiarjties whose discussion will be left to the reader.

Polar coordinates $r, \theta$ can at once be introduced, with

$$
x=r \cos \theta, y=r \sin \theta
$$

as their relations to the previous orthogonal $x, y$. The use of such coordinates amounts to writing, for the position vector of any point of the plane,

$$
\mathbf{r}=r(\mathbf{i} \cos \theta+\mathbf{j} \sin \theta), \quad . \quad . \quad .
$$

where $i, j$ is a pair of orthogonal unit vectors.

6. Extension to Three-Space.-In order to extend the preceding investigation to three-dimensional space let us take, instead of $\kappa$, a non-ruled closed quadric (ellipsoid), surrounding the origin $O$, as the standard or unit surface. We will denote it shortly by $Q$. The $T$-plane will be the polar plane of $O$ with respect to $Q$.

The equation of $Q$ with any non-coplanar triad a, b, c as axes will be

$$
x^{2}+y^{2}+z^{2}+2 \alpha_{23} y z+2 a_{31} z x+2 a_{12} x y=1 \text {. }
$$


The plane section $z=0$, and therefore any diametral section * of $Q$, will be a conic whose equation will be of the form

$$
x^{2}+y^{2}+2 a_{12} x y=1 \text {. }
$$

This will play, in the said plane, the part of our previous $x$. Thus, defining ab as the corresponding coefficient of $2 x y$ and reasoning as before, we shall obtain the scalar product $\mathbf{A B}$ with all its properties, provided that $\mathbf{A}, \mathbf{B}$ are in a diametral plane. But. if $\mathbf{A}, \mathbf{B}$ are not in a diametral plane we can construct vectors equal to them in an appropriate plane, viz. that passing through $O$ and the straight $T_{\mathrm{A}} T_{\mathrm{B}}$. Moreover, we can draw these, and all olher vectors, from $O$ as origin. It is enough to declare, as in the case of two dimensions, that $\mathbf{A}^{\prime} \mathbf{B}^{\prime}=\mathbf{A B}$ whenever $\mathbf{A}^{\prime}=\mathbf{A}$ and $\mathbf{B}^{\prime}=\mathbf{B}$, no matter where $\mathbf{A}^{\prime}, \mathbf{B}^{\prime}$ are drawn.

All fundamental questions are thus reduced to those treated previously, and need not detain us here.

It remains only to show how triads of mutually orthogonal vectors, reducing the $Q$-equation to $x^{2}+y^{2}+z^{2}=1$, can be constructed. And, by what has just been said, we can without loss to generality, confine our attention to rectors drawn from $O$ as origin.

Let, therefore, $a=O_{a}$ be any given unit vector, and $T_{\alpha}$ its terminus, $i . e$. the cross of the a-line with the 'T-plane. The second vector $b$ normal to a being required to be in a given plane, lay that plane through $O$ and draw from $T_{a}$ a tangent to $\kappa_{a b}$, the section of $Q$. The contact point $b$ will be the end-point of the required $b$. ('The other tangent will give $\mathbf{b}^{\prime}=-\mathrm{b}$, as before.) It remains to construct a third vector $\mathrm{c}$ such that $\mathbf{c a}=0$ and $\mathbf{c b}=0$. Lay through $T_{a} T_{b}$ a plane tangent to $Q$. Then its contact point will be the end-point $c$ of the required unit vector $c$.

To verify this statement notice that if $\mathbf{c}$ is to be normal to $\mathbf{a}, \mathbf{b}$, which are already normal to one another, the equation of $Q$ with respect to $a, b, c$ as axes will be $x^{2}+y^{2}+z^{2}=1$, and therefore the equation of the plane touching it at any point $\xi, \eta, \zeta$ will be easily found to be

$$
\xi \cdot x+\eta y+\zeta z=1,
$$

so that the tangent plane at $c(\xi=1, \eta=\zeta=0)$ should simply be $x=1$. Now, this is precisely the plane through $c, T_{a}, T_{b}$.

The second tangent plane through $T_{a} T_{b}$ will touch $Q$ in $c^{\prime}$, the end-point of the vector -c.

Thus any number of orthogonal triads $a, b, c$ can be

* I. e. by a plane passing through $O$. 
constructed *. We shall henceforth denote any such triad of normal unit vectors by $i, j, k$, assuming this to be their order in a right-handed system.

It may be interesting to notice that the end-point $P$ of the vector

$$
\mathbf{P}=\mathbf{i}+\mathbf{j}+\mathbf{k} \text {. . . . . . }
$$

is the cross of the three tangent planes to $Q$ at the endpoints $i, j, k$ of these normal vectors. More generally, if $\mathbf{a}, \mathbf{b}, \mathbf{c}$ are any three unit vectors "equally inclined" to one another, $i$. e. such that $a b=b c=c a=\lambda$, the planes touching $Q$ at $a, b$, c cross in the end-point of the vector

$$
\mathbf{P}=\frac{\mathbf{a}+\mathbf{b}+\mathbf{c}}{1+2 \lambda} \ldots . \quad \cdot \quad \cdot \quad \cdot
$$

The proof, similar to that of equation (2), offers no difficulty.

All other details concerning the passage from a plane to three-space may be left to the care of the reader. He will easily convince himself that the distributive property,

$$
\mathbf{A}(\mathbf{B}+\mathbf{C})=\mathbf{A B}+\mathbf{A C},
$$

with its consequences, will remain valid, whether $\mathbf{A}, \mathbf{B}, \mathbf{C}$ are (or can be made) coplanar or not.

One of the consequences will be the generalized Pythagorean theorem for three dimensions,

$$
D^{2}=\mathbf{D}^{2}=\mathbf{A}^{2}+\mathbf{B}^{2}+\mathbf{C}^{2},
$$

where $\mathbf{A}, \mathbf{B}, \mathbf{C}$ are any normal vectors and $\mathbf{D}$ their sum. Thus also the line-element, $i$. e. the tensor $d s$ of the vector

will be given by

$$
d \mathbf{r}=\mathbf{i} d a+\mathbf{j} d y+\mathbf{k} d z
$$

$$
d s^{2}=d x^{2}+d y^{2}+d z^{2}
$$

the extension of (14) to space. The introduction of polar coordinates and allied questions do not call for any. explanations.

7. Vector Product of two Vectors.-Once in possession of the concept of orthogonality we can define the vector product of two vectors in much the same way as in common vector algebra.

Let $\mathbf{A}=A \mathrm{a}$ and $\mathbf{B}=B \mathbf{b}$, and let $\boldsymbol{\theta}$ be the concave angle

* In technical language, $O T_{a} T_{b} T_{c}$ will be a self-polar tetrahedron with respect to $x$. 
(i.e. not exceeding $\pi$ ) made by $\mathrm{a}, \mathrm{b}$. Then we call vectorproduct of $\mathrm{A}$ into $\mathrm{B}$ and denote $\mathrm{by}$

$$
\mathbf{C}=\mathrm{VAB} \text {, }
$$

a third vector whose tensor is $C=A B \sin \theta$, drawn perpendicularly to $a, b$ and so that $a$ right-handed rotation abont $c$ carries the vector a to $b$.

From this definition it follows at once that

$$
\mathrm{VAB}=-\mathrm{VBA}=A B \mathrm{Vab} \text {. }
$$

Further, if $\mathbf{A}, \mathbf{B}$ are coterminal vectors, $\mathrm{VAB}=0$ (corresponding to the common case of "parallel" vectors). In particular, for our normal, right-handed triad of unit vectors $\mathbf{i}, \mathbf{j}, \mathbf{k}$

$$
\text { Vii }=0, \text { etc. } \quad V \mathbf{i j}=\mathbf{k}, \quad V \mathbf{j k}=\mathbf{i}, \quad V \mathbf{k i}=\mathbf{j} .
$$

The distributivity of this vector product can be proved in a variety of ways. The shortest and easiest seems to be the following proof. Let

$$
A=A_{1} i+A_{2} j+A_{3} k,
$$

and similarly for $\mathbf{B}$ and $\mathbf{C}$. Then, $\mathbf{C}$ being normal to $\mathbf{A}, \mathbf{B}$, by definition, we have

$$
\begin{aligned}
& \mathrm{CA}=\mathrm{C}_{1} \mathrm{~A}_{1}+\mathrm{C}_{2} \mathrm{~A}_{2}+\mathrm{C}_{3} \mathrm{~A}_{3}=0, \\
& \mathbf{C B}=\mathrm{C}_{1} \mathrm{~B}_{1}+\mathrm{C}_{2} \mathrm{~B}_{2}+\mathrm{C}_{3} \mathrm{~B}_{3}=0,
\end{aligned}
$$
whence, solving these two equations for the ratios $\frac{C_{1}}{C_{3}}, \frac{C_{2}}{C_{3}}$,
we find at once

$$
\begin{array}{r}
C_{1}=\lambda\left(A_{2} B_{3}-A_{3} B_{2}\right), C_{2}=\lambda\left(A_{3} B_{1}-A_{1} B_{3}\right), \\
C_{3}=\lambda\left(A_{1} B_{2}-A_{2} B_{1}\right), \quad . \quad . .
\end{array}
$$

where $\lambda$ is a scalar. Remembering that, by definition,

$$
\mathrm{C}^{2} \equiv \mathrm{C}_{1}{ }^{2}+\mathrm{C}_{2}{ }^{2}+\mathrm{C}_{3}{ }^{2}=\mathrm{A}^{2} \mathrm{~B}^{2} \sin ^{2} \theta,
$$

we have $\lambda^{2}=1$, that is $\lambda= \pm 1$. To decide the sign take, for instance, $\mathbf{A}=\mathbf{i}, \quad B=\mathrm{j}$. Then, by definition, $\mathbf{C}=\mathbf{k}, i$. e. $C_{1}=C_{2}=0, C_{3}=1$; and since $A_{1}=B_{2}=1$, while $A_{2}, B_{1}$, etc. all vanish, we have, by $\left(18^{\prime}\right), \lambda=1$. Thus the expanded form of the vector product (defined intrinsically at the outset) is

$V A B=i\left(A_{2} B_{3}-A_{3} B_{2}\right)+j\left(A_{3} B_{1}-A_{1} B_{3}\right)+k\left(A_{1} B_{2}-A_{2} B_{1}\right),(18)$.

familiar from ordinary vector algebra. Now, if $\mathbf{M}$ be any vector, the components of $B+\mathbf{K}$ along $i, j, \mathbf{k}$ are (by the 
distributive property of scalar multiplication of vectors) $B_{1}+M_{1}$, etc. Thus

$$
\mathrm{VA}(\mathbf{B}+\mathbf{M})=\mathrm{i}\left[A_{2}\left(B_{3}+M_{3}\right)-A_{3}\left(B_{2}+M_{2}\right)\right]+\text { etc., }
$$

and this is the same thing as

$$
\mathrm{VA}(\mathbf{B}+\mathbf{M})=\mathrm{VAB}+\mathrm{VAM}, \quad . \quad .
$$

the distributive property of the vector product.

This being valid, all other properties of such products and of mixed producis, AVBC, etc., known from ordinary vector algebra, will continue to hold. It is therefore needless to dwell upon them any further. It will be enough to notice that the so-called "parallelepipedal" property,

$$
A V B C=B V C A=C V A B \text {, }
$$

which is commonly proved by saying that each of these three expressions represents the "volume" of the parallelepipedon A, B, C (right-handed), will in our case be better proved without the aid of the concept of "volume," since we have not defined any such concept. The vector VBC being expanded as in (18), we see at once that its scalar product into $\mathrm{A}$ is $A_{1}\left(B_{2} C_{3}-B_{3} C_{2}\right)+$ etc., or

$$
\operatorname{AVBC}=\left|\begin{array}{lll}
A_{1} & A_{2} & A_{3} \\
B_{1} & B_{2} & B_{3} \\
C_{1} & C_{2} & C_{3}
\end{array}\right|,
$$

and this is, by the well-known property of determinants, the same thing as BVCA or CVAB. Q.E.D.

The vector multiplication of two vector polynomials will be handled as the scalar multiplication, the only difference being that the order of fictors must be preserved or, if inverted, the sign of the purtial product must be changed, the well-known rule of orkiniry vector algebra.

8. General remarks.-Thus, hatving defined vector addition and vector multiplication with reference to a conventional $T$-line and a $\cdot$ standard conic $\kappa$ (or $J$-plane and quadric $Q$ ), we have seen that the resulting Vector Algelora and differential analysis are formally identical with ordinary, euclidean Vector Algebra and Analysis (the only difference being that inches, etc. are replaced by staudtian, and that the angle concept is modified and generalized). Does this mean thiat we have involuntarily lapsed into euclidean space? By no means. We have only set up, in the general projective space, a euclidean or a parabolic system of measurement. In faet, that our vector algebra is entirely equivalent to parabolic metrics in the sense of Cayley-Klein, will be seen at 
once by noticing that the $T$-points or termini of our pairs of orthogonal lines (such as $T_{i}, T_{j}$ ) form on the $T$-line an involution, with imaginary double points*. Now, this with the rôle played throughout by the $T$-line is the well-known characteristic of the parabolic system of measurement ("absolute involution," "circular points"). Only the way in which it was here set $u p$ is, if I may judge, a much more natural one and easier to lollow than that based on the usual application of Cayley's ideas.

Such, however, being the nature of the measurement system equivalent to the proposed Vector Algebra, it is important to notice that it can be set up and used as well in Euclid's as in Lobatchevsky's or Riemann's (elliptic) space, i.e. no matter whether Euclid's parallel-axiom is valid or whether there is a whole pencil of non-intersecting lines, or whether there are none at all. (In order to avoid confusion, it would be well to speak in this connexion of euclidean, lobatchevskyan, and riemannian spaces and to reserve the names parabolic, hyperbolic, and elliptic to the metrical systems themselves.)

To make these remarks more plain let us take the case of two dimensions, which will suffice, and let us imagine an ordinary spherical surface in ordinary euclidean space. In order to avoid antipodal complications take an appropriately limited portion $\sigma$ of this surface and consider only such figures which, together with all their auxiliaries, do not surpass the limits of $\sigma$. Let the geodesies of $\sigma$ (great circles) be substituted for the straight lines of our fundamental definitions (P.V.A.). Thus an arrowed segment $O X$ of such a line will be called a vector, $\mathbf{X}$. One of these geodesics being chosen as the $T$-line, the sum $\mathbf{X}+\mathbf{Y}$ will be defined as the vector whose origin is $O$ and whose end-point is the cross of the geodesics $X T_{y}$ and $Y T_{x}$. Thus vector addition will be commutative. And since Desargues' theorem is obviously valid for spherical triangles in perspective $t$, the addition of

* The easiest way to see this is to draw from $O$, the centre of $\kappa$, a pencil of lines $l$ and to cut it by any transveisal $s$. Let $A$ be the foot of the normal $h$ drawn from $O$ to $s$, and let a line $l$ and its corresponding (orthogonal) $l^{\prime}$ cut $s$ in $L$ and $L$, respectively. Then, writing $x^{\prime}$ and $-x$ for the coordinates of $L^{\prime}, L$ with respect to $A$, we have $x x^{\prime}=-h^{2}$, by the genelalized Pythagorean theorem. Whence also the correspondence $l, l$ in the pencil of lines is seen to be an involution of the said kind.

+ As far as I can gather this property is not mentioned in text-books, but it is, none the less, a most immediate consequence of the well-linown thecrem on perspective trihedra. The usual theorem of Desargues is obtained by taking of the space figure a plane section. In order to obtain the above theorem, it is enough to intersect it with our spherical surface. 


\section{Further Contributions to Non-Metrical Vector Algebra.}

these vectors will also be associative (P.V.A., p. 8), that is to say, $(\mathbf{X}+\mathbf{Y})+\mathbf{Z}=\mathbf{X}+(\mathbf{Y}+\mathbf{Z})$. Thus all addition rules will continue to hold, together with their consequences. among which is the construction of the staudtian scale, and so on. Again, "conics" can be generated on $\sigma$, as loci of crosses of correlated lines of two projective pencils, say by the process

$$
\mathbf{R}=[\mathbf{X}+\mathbf{Y}\rceil_{t},
$$

as explained in P.V.A., Section 15. One such closed conic $\kappa$ can be drawn round $O$ and used as a standard conic, and the whole line of reasoning given above can be literally repeated, leading to the definition of orthogonal lines and thence to the concept of the scalar product of two vectors. This product will again obey the distributive law so that, for instance, the generalized theorem of Pythagoras, $\mathbf{C}^{2}=\mathbf{A}^{2}+\mathbf{B}^{2}$, will continue to hold for " right-angled" spherical triangles, and so on. In short, we shall have an ordinary vector algebra, or a parabolic measurement system, set up upon the contemplated portion of the spherical surface. On the same surface we can also set up a hyperbolic system (by taking one of the said real "conics" as Cayley's "absolute" and adopting his definition of distance and of angle) or an elliptic system of measurement, which latter will simply be the usual spherical trigonometry. In the last two cases we are deprived of the facilities of an associative and distributive vector algebra, while in the first case we have a vector algebra consisting of addition and scalar multiplication formally identical with ordinary vector algebra.

The same remarks and explanations apply, mutatis mutandis, to the case of three-dimensional space, where also the vector product of vectors comes to its rights.

9. Supplementary Constructions to Section 5. - It has seemed advisable to add a few words about the constructions involved in what was treated in Section 5, and in particular on p. 132.

$A$. Given an angle $\alpha=(\mathrm{a}, \mathrm{b})=a O b$, say with the centre of $\kappa$ as vertex, to constmuct an angle $\alpha^{\prime}=\alpha$ with any given $O^{\prime}$ as vertex and $l$ as one of its sides, coplanar with $\mathrm{a}, \mathrm{b}$.

Let $T_{l}$ be the terminus of $l$. Draw the join $T_{l} a$ cutting $\kappa$ again in $c$. Double the angle $\alpha$ in the explained way, making $a O d=2 \alpha$. Join $c d$ cutting the $T$-line in $T^{\prime \prime}$. Then $O^{\prime} 7^{\prime \prime}$ will be the required second side of $\alpha^{\prime}=\alpha$. In fact, the angle acd is $2\left(\frac{\alpha}{2}\right)=\alpha$; the termini of the sides of this angle are $T_{l} T^{\prime} ;$ hence, $T_{l} O^{\prime} T^{\prime} \equiv \alpha^{\prime}=\alpha$. 
An explanatory figure is scarcely necessary. The proof just given is based upon the lemma : All angles $\beta$ subtended by the arc ad of the "central" angle $a O d$ and having their vertices on $\kappa$ are equal to $\frac{1}{2} a O d$. The proof of this lemma, a generalization of the familiar theorem on peripheral angles, offers no difficulties, and may be left to the reader as a simple exercise in vector algebra. The generalization of the above construction to non-coplanar $\alpha^{\prime}, \alpha$ is again recommended to the reader.

$B$. Any vector $\mathbf{R}=O R$ being given, construct upon a given ray $O T_{s}$ the vector $\mathbf{S}=O S$, whose tensor is equal to that of $\mathbf{R}$, in symbols,

$$
|\mathbf{S}|=|\mathbf{R}|,
$$

or in (generalized) familiar language, construct a segment OS congruent to $\mathrm{OR}$.

The vector $\mathrm{r}=\mathrm{O} r$ being the unit of $\mathrm{R}$, and $\mathrm{s}=\mathrm{O} s$ the unit of the required $\mathbf{S}$, draw the join $s r$ up to its terminus ' $\mathrm{T}$ '. Then $T^{\prime} R$ will cut $O T_{s}$ in the end-point $S$ of the required vector.

'Thus also we can easily draw a conic $\kappa_{\mathrm{R}}$, homologous to $\kappa$, through any given point $R$. In fact, all rays centred at the point just called $T^{\prime}$ will cut the r-line and the s-line in points $M, N$, such that $|O N|=|O M|$, or in other words, in points $M, N$ lying on the same conic homologous with $\kappa$. The extension to non-coinciding origins or centres offers no difficulty.

London, March 12th, 1919, Research Dept., Adam Hilger, Ltd.

\author{
X. Electromagnetic Waves. \\ By T. J. I'A. BromwICH, Sc.D., F.R.S.* \\ Introductory Summary.
}

$r$ HE following pages contain a general solution of the 1 electromagnetic equations of wave-propagation : in $\$ 1$ these formulæ are expressed in terms of general orthogonal coordinates, and in $\$ 2$ it is proved that these formulæ are the most general possible-at any rate for spherical polar coordinates.

It naturally follows that the solution of $\S 1$ should include all known types of solution; and this fact is confirmed by detailed examination. In $\S 3$ the spherical polar solntion

* Communicated by the Author. 